\title{
Prostate Acinar Adenocarcinoma, Sarcomatoid Variant
}

National Cancer Institute

\section{Source}

National Cancer Institute. Prostate Acinar Adenocarcinoma, Sarcomatoid Variant. NCI

Thesaurus. Code C5530.

A rare morphologic variant of acinar adenocarcinoma of the prostate gland with unfavorable prognosis, composed of both malignant glandular and sarcomatous components. The sarcomatous component contains a malignant spindle cell proliferation or specific mesenchymal elements including osteosarcoma, chondrosarcoma, and leiomyosarcoma. 